\title{
Retención dental por diente supernumerario - Caso Clínico
}

Dental retention for supernumerary tooth - Clinical case

\author{
Melissa I. Oviedo-Viveros ${ }^{a}$, Carmen Delgadillo-Balderas ${ }^{b}$, Ana K. Bazán-Suárez, Eva M. \\ Molina-Trinidad ${ }^{d}$, Marco A. Becerril-Flores ${ }^{e}$.
}

\begin{abstract}
:
Supernumerary teeth are additional teeth that develop in the jaws. In the upper anterior sector, when they are not erupted, they are a frequent cause of alterations of the eruption of permanent teeth. The diagnosis and treatment of these dental organs is necessary to avoid subsequent complications that could alter the dental arch. This is a clinical case of a 10 -year-old girl who presented with retention of the central maxillary incisor related to a supernumerary tooth. The proposed treatment involved the surgical extraction of the supernumerary tooth, a waiting and observation approach, the surgical exposure of the retained central incisor and the orthodontic traction thereof with a removable device.
\end{abstract}

Keywords:

Supernumeraries, rash, retention, traction

\section{Resumen:}

Los dientes supernumerarios son dientes adicionales que se desarrollan en los maxilares. En el sector anterosuperior, cuando no están erupcionados, son causa frecuente de alteraciones de la erupción de los dientes permanentes. El diagnóstico y tratamiento de estos dientes se hace necesario para evitar complicaciones posteriores que pudieran alterar el arco dental. Este es un caso clínico de una niña de 10 años que se presentó con retención del incisivo central maxilar relacionado con un diente supernumerario. El tratamiento propuesto implicó la extracción quirúrgica del diente supernumerario, un enfoque de espera y observación, la exposición quirú rgica del incisivo central retenido y la tracción de ortodoncia del mismo con un dispositivo extraíble.

\section{Palabras Clave:}

Supernumerarios, erupción, retención, tracción

\section{Introducción}

La hiperdoncia o dientes supernumerarios es una anomalía de origen desconocido que se caracteriza por un aumento en el número de dientes, los cuales rompen el esquema numérico de la dentición normal; es decir, que no tendrían que haber aparecido, pero que se desarrollaron adicionalmente al número normal de dientes que forman la dentadura, ocupando un lugar que no les corresponde. ${ }^{1}$

Se suelen detectar casualmente al realizar una radiografía de rutina o como consecuencia de la exploración clínica para averiguar la causa por la cual un diente permanente no ha erupcionado. ${ }^{2}$ Es una condición que habitualmente no produce síntomas y sólo un $25 \%$ de los dientes supernumerarios llegan a erupcionar con normalidad, y en caso de no lograrlo, pueden causar

\footnotetext{
a Autor de Correspondencia, Universidad Autónoma del Estado de Hidalgo, Instituto de Ciencias de la Salud, https://orcid.org/0000-0001-6290-7984, Email: melissa18itzel@gmail.com

${ }^{\text {b }}$ Autor de Correspondencia, Universidad Autónoma del Estado de Hidalgo, Instituto de Ciencias de la Salud, https://orcid.org/0000-0002-200-5549, Email: carmenbd@uaeh.edu.mx

• Universidad Autónoma del Estado de Hidalgo, Instituto de Ciencias de la Salud, https://orcid.org/0000-0002-2400-5549, Email: karenbazann@ gmail.com d Universidad Autónoma del Estado de Hidalgo, Instituto de Ciencias de la Salud, https://orcid.org/0000-0001-9654-5918, Email: emolinatrinidad@ gmail.com e Universidad Autónoma del Estado de Hidalgo, Instituto de Ciencias de la Salud, https://orcid.org/0000-0002-2322-4686, Email: mbecerril_65@yahoo.com
} 
importantes problemas en el desarrollo normal de la dentición, originando maloclusiones importantes, alteración de la estética de la sonrisa cuando se ubican en la parte central del arco dental, y retención del diente permanente, lo que puede causar la formación de un quiste dentígero. Derivado de esto, un diagnóstico precoz de esta situación es de suma importancia para evitar futuras complicaciones. ${ }^{3}$

La etiología de la hiperdoncia es desconocida, aunque su origen podría estar relacionada en los fenómenos de inducción y diferenciación de la lámina dental durante las primeras fases de la formación dentaria $^{4}$ y/o por una avulsión accidental del folículo dentario. ${ }^{5}$

La presencia de dientes supernumerarios es más frecuente en hombres que en mujeres y tiene una predilección por el maxilar superior en un $90-98 \%{ }^{2}$ La hiperdoncia más frecuente es el canino con una prevalencia del $6 \%$, seguido por el incisivo central en un $0.06 \%$ a $0.2 \%$, este diente erupciona normalmente entre los 8 a 10 años y al haber una erupción tardía provoca un efecto adverso sobre la estética, la función y el habla, también puede provocar migración de dientes adyacentes, pérdida de espacio y desviación de la línea media.

Por ello es importante realizar una inspección durante el desarrollo de la dentición mixta para evaluar si existe alguna anomalía como la erupción dental tardía, esta evaluación consiste en contar e identificar los órganos dentales presentes, tanto en forma clínica como radiográfica y así poder diagnosticar en un estadio temprano las anomalías para poder establecer un diagnóstico definitivo y un adecuado plan de tratamiento en base a la edad, género, etiología, prevalencia, y localización de los órganos dentales supernumerarios. ${ }^{8}$

\section{Presentación del Caso}

Paciente femenino de 10 años derivada a las clínicas odontológicas pertenecientes a la Universidad Autónoma del Estado de Hidalgo con la queja principal de un diente frontal izquierdo no erupcionado.

El examen clínico reveló la ausencia del incisivo central superior izquierdo (Figura 1). La radiografía panorámica confirmó la retención del incisivo central superior izquierdo con orientación normal, por la obstrucción con un diente supernumerario ubicado en un espacio entre el diente número 21 y 22 (Figura 2).

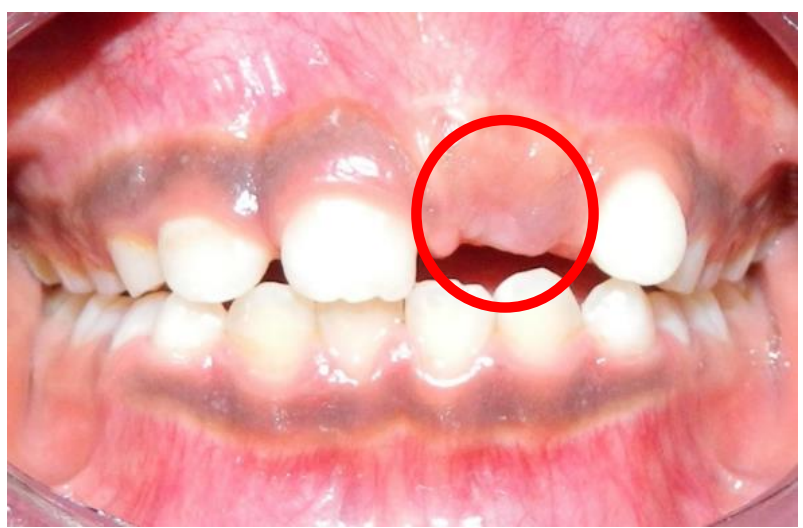

Figura 1. Órgano dental número 21 ausente clínicamente.

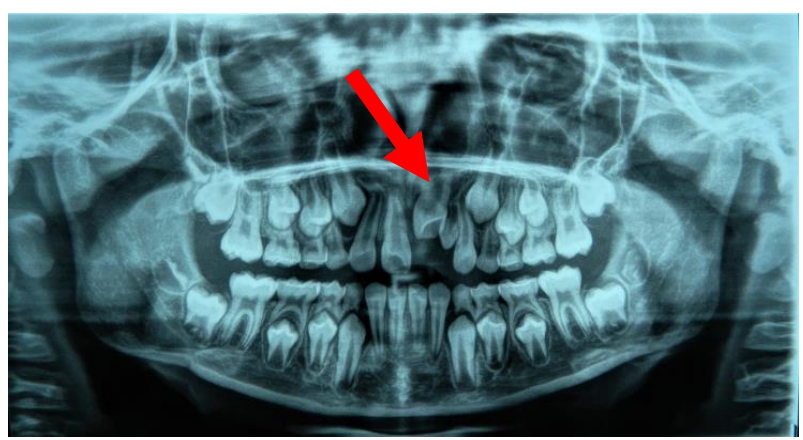

Figura 2. Radiografía panorámica. Órgano dental número 21 retenido por obstrucción de un diente.

Objetivos del tratamiento:

1. Extracción quirúrgica del diente supernumerario.

2. Exposición de la corona del incisivo central superior izquierdo.

3. Un enfoque de esperar y observar la erupción espontánea del diente retenido.

4. Tracción del diente retenido con un dispositivo de ortodoncia.

Secuencia de tratamiento:

Se ingresó a la paciente a clínica de cirugía bucal para hacer el tratamiento correspondiente para extraer el diente supernumerario (Figura 3).

Posterior a la extracción del diente supernumerario se le dieron las indicaciones posquirúrgicas establecidas al paciente para su correcta cicatrización y recuperación, así como se le sugirió que, consumiera alimentos de consistencia fibrosa que ayudaran a estimular la masticación y por ende la erupción del diente retenido. 


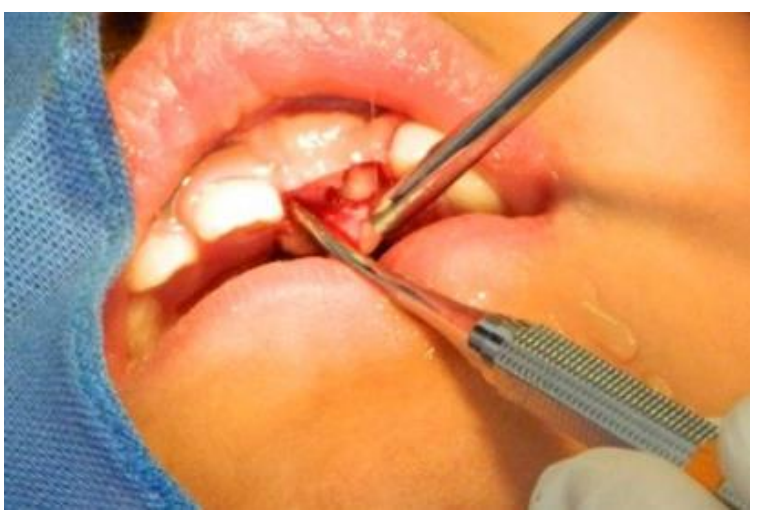

Figura 3. Diente supernumerario expuesto quirúrgicamente.

Meses después de la extracción del diente supernumerario, el diente número 21 aun no erupcionaba pese a que radiográficamente se observa que había avanzado en la guía de erupción (Figure 4). Derivado de esto, se decide hacer la exposición quirúrgica del elemento retenido para su posterior tracción ortodóntica (Figure 5 y 6).

Para ello, se realizó la exposición quirúrgica del diente número 21 y se comienza a hacer el diseño de un aparato de ortodoncia removible para poder traccionar el diente hacia su adecuada posición en el arco dental.

Una vez que se realiza la exposición del diente, se cementa un botón en la cara vestibular del mismo y se coloca un aparato de ortodoncia removible de base acrílica cubriendo el paladar duro y alambre de acero inoxidable con ganchos circunferenciales en los primeros molares permanentes y ganchos de bola entre los molares deciduos, así como un gancho adicional para sujetar la liga el cual se une al botón de la cara vestibular del diente 21 , aplicando una fuerza de tracción ligera para posicionar el diente en el arco.

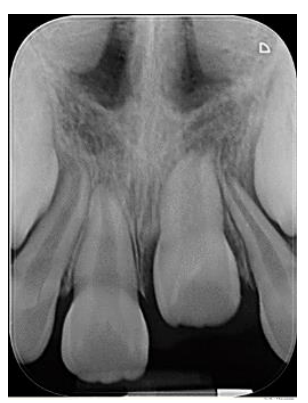

Figura 4. Radiografía periapical de incisivos dentales superiores 2 meses después de la extracción del diente supernumerario.

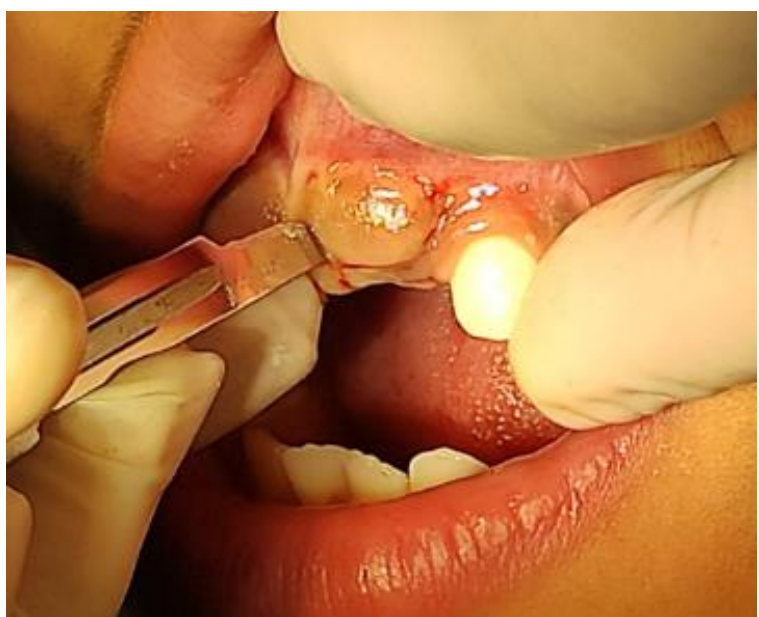

Figura 5. Incisión liberatriz del diente número 21.

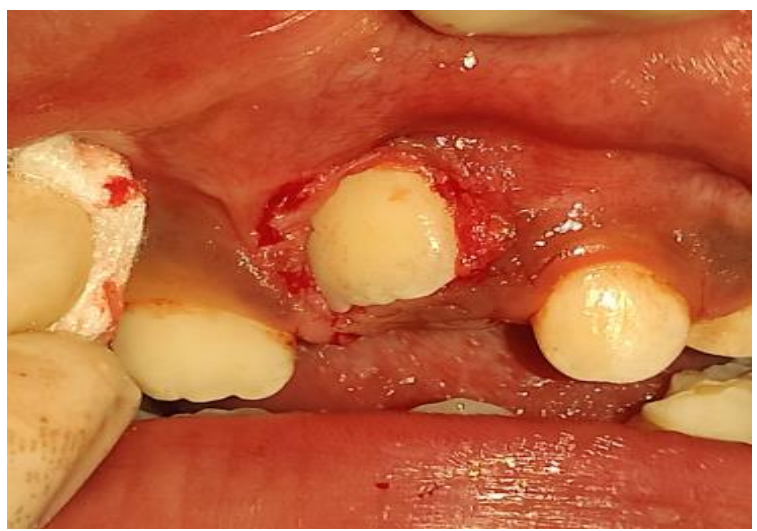

Figura 6. Exposición del diente número 21.

Se utilizaron ligas de fuerza ligera de $2 \frac{1}{2} \mathrm{oz}$, las cuales fueron medidas con un dontrix para medir la fuerza de tracción que se estaría aplicando con el uso del aparato removible, se le dieron instrucciones de uso al paciente y a sus padres, y se recomendó utilizar el aparato mínimo 16 horas al día (Figura 7).

Se mantuvo el uso del aparato de ortodoncia removible durante los siguientes 3 meses, en los cuales se valoraba al paciente en una cita por semana para determinar que la fuerza empleada no fuese excesiva en el transcurso del tratamiento $e$ ir determinando los avances que se tenían del mismo.

\section{Discusión}

Este tipo de casos principalmente es notorio y preocupan a los padres en la dentición mixta temprana debido a problemas estéticos y funcionales, así como secuelas psicológicas en los niños. ${ }^{7}$ Las opciones de tratamiento incluyen extracción de los dientes supernumerarios, observación y exposición quirúrgica del diente retenido. ${ }^{8}$ Muchos autores han descrito diferentes enfoques para esta situación. Sin embargo, se debe elegir en base al 
diagnóstico presuntivo basado en elementos de diagnóstico. Si no se produce una erupción espontánea, la elección quirúrgica es la exposición del diente y la tracción ortodóncica de los dientes retenidos.

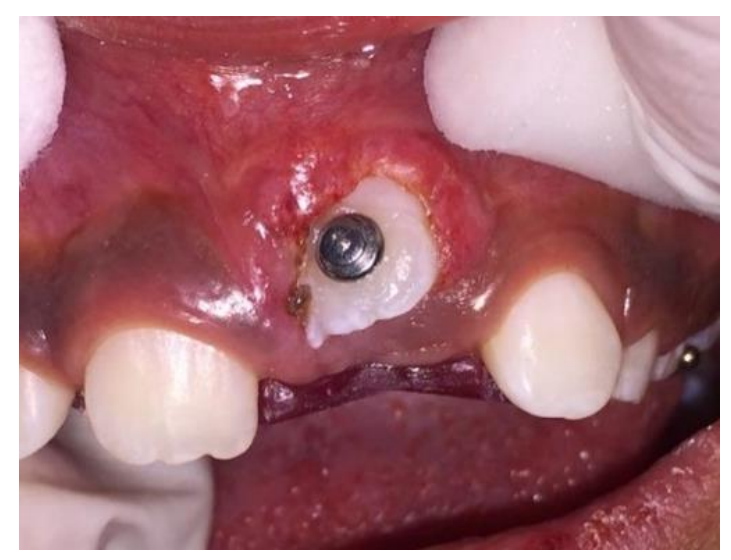

Figura 7. Cementación del botón en la cara vestibular del órgano dental 21

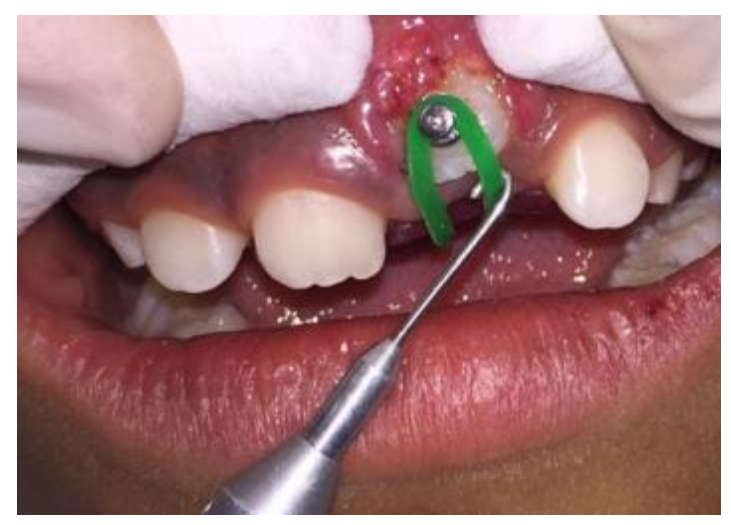

Figura 8. Medición con dontrix de la fuerza de tracción aplicada.

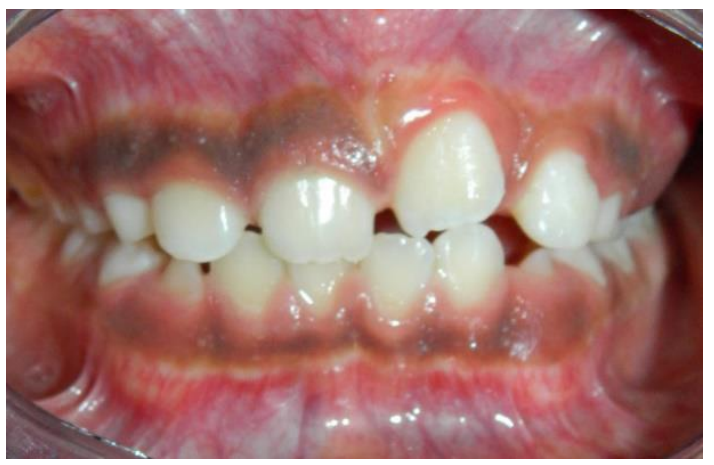

Figura 9. Avances después de 3 meses de uso del aparato de ortodoncia removible.

\section{Conclusiones}

Es importante diagnosticar la presencia de dientes supernumerarios, tanto en forma, número y posición, así como clínica y radiográficamente, antes de realizar el diagnóstico definitivo y elaborar un plan de tratamiento. El tratamiento de un supernumerario, normalmente, es la extracción, por motivos estéticos y funcionales, para evitar las posibles complicaciones en la etapa de erupción de la dentición mixta.

\section{Referencias}

[1] Sanitas. Clínicas Dentales [Internet]. 2020. p. 1-8. Disponible en: https://www.sanitas.es/sanitas/seguros/es/particulares/biblioteca-desalud/salud-dental/dientes-supernumerarios.html

[2] Jiménez Y, Coca R, Dúran D. Dientes supernumerarios y retención múltiple. Revisión de la literatura y presentación de un paciente. Acta Médica del Cent. 2017;11(2):58-63.

[3] Sierra M, Peral A, de Lara L, Brito P, Bonilla E. Manejo de paciente con retención de incisivo central permanente causada por mesiodens. $: 410-4$.

[4] Ramón R, Miegimolle M, Gallegos L. Anomalías dentarias de número: hiperdoncia/hipodoncia. A propósito de un caso. Odonto Pediátrica. 2014;2214223(3):209-15.

[5] Brenes J. Dientes supernumerarios: presentación de un caso clínico. Rev Científica Odontológica. 2009;5(2):58-62.

[6] Oropeza M. Dientes supernumerarios. Reporte de un caso clínico. Rev Odontológica Mex [Internet]. 2013;17(2):91-6. Disponible en: http://dx.doi.org/10.1016/S1870-199X(13)72022-6

[7] Colombo E, Quiróz O. Problemas asociados con la presencia de dientes supernumerarios - Reporte de caso. Rev Latinoam Ortodoncia y odontopediatría [Internet]. 2013;8-10. Disponible en: https://www.actaodontologica.com/ediciones/2015/suplemento/art77/\#

[8] Mohammad-Rabei E, Shamsi A, Farahani M. Treatment of Impacted Maxillary Central Incisor with Removable Appliance: A Case Report. Int J Med Pharm Case Reports. 2016;8(2):1-6. 